\title{
Role of hemi-epiphysiodesis using 8-plate system in the correction of coronal plane deformities around the knee
}

\author{
K Venkataramana ${ }^{1}$, Yarram Nagarjuna Reddy ${ }^{2, *}$, Jagadesh Gudaru ${ }^{3}$, K. Deepak ${ }^{4}$ \\ ${ }^{\mathbf{1}}$ Assistant Professor, ${ }^{2}$ Resident, ${ }^{3}$ Director and HOD, ${ }^{4}$ Associate Professor, Dept. of Orthopaedics, Balaji Institute of Surgery, \\ Research and Rehabilitation for the Disabled (BIRRD), Andhra Pradesh, India
}

*Corresponding Author:

Email: nagarjunareddyy $2 @$ gmail.com

\begin{abstract}
Introduction: Angular deformities around the knee in children is a common finding, these deformities are from diverse group of etiologies. Most of these deformities are physiological in nature corresponding to the changes in the alignment of tibio-femoral angle from birth to adolescence. Angular deformities can also be developed due to pathological causes like skeletal dysplasias, metabolic bone disorders, Blount's disease and trauma. Osteotomies were the mainstay of treatment for correcting deformities in both adults and children been replaced by Hemiepiphy siodesis using 8 plates having less complication rate.

Materials and Methods: A total of 49 knees (35 genu valgum, 14 genu varum) of 30 patients were studied. 8 -plates were used for correction of coronal plane deformities around the knee. All cases were followed for minimum period of 12 months. Preoperative clinical and radiological measurements were compared to post-operative measurements.

Results: There was statistically significant improvement in clinical and radiological outcome measures. In genu valgum group preoperative mean Tibio-femoral angle (TFA) improved from 24.16 to 8.36. Mean Mechanical axis deviation (MAD) improved from 4.26 to 1.09 . Rate of correction of TFA is $1.1 /$ month, rate of correction of MAD is $0.22 /$ month. In genu varum group preoperative mean TFA improved from 34.58 to 21.47 . Mean MAD improved from 4.92 to 0.63 . Rate of correction of TFA is $0.77 /$ month, rate of correction of MAD is 0.12 /month.

Conclusion: Hemi-epiphysiodesis using 8-plate systemin the correction of coronal plane deformities around the knee is a safe and effective procedure.
\end{abstract}

Keywords: Hemiepiphysiodesis, Guided growth modulation, Genu varum, Genu valgum.

\section{Introduction}

Angular deformities around the knee are from diverse group of etiologies, which make management of these deformities a great challenge. Most of these deformities are physiological in nature corresponding to the physiological changes in the alignment of tibiofemoral angle from birth to adolescence. ${ }^{1,2}$ Most of the children are bow legged at birth, become knock-kneed by 2 years of age; this knock-knee alignment peaks by 4 years and then improves by age 8.Most of the children improve spontaneously to the normal adult femorotibial angle before the age of 8 years. ${ }^{1}$ Due to Idiopathic reasons, skeletal dysplasias, metabolic bone disorders, Blount's disease, trauma and extremes of physiological variations, long bones of the leg may grow out of normal tibio-femoral alignment. These angular deformities around the knee alter the biomechanics and cause distorted stress distribution. Pain, limitation of activity, joint instability, growth disturbance may develop in significant number of children, in whom physiological correction of bow legs and knock knees doesn't occur. Abnormal or injured physis may result in wide variety of problems that require orthopedic intervention. Majority of the physiological deformities correct themselves by adolescence requiring no treatment. However some of these physiological deformities may persist beyond adolescence necessitating orthopedic intervention. Patients with pathological deformities require early intervention. ${ }^{3}$ Traditionally osteotomies are the mainstay of treatment for correcting deformities in both adults and children. However, the associated soft tissue dissection, compartment syndrome, infection, neurovascular injuries, delayed union, malunion, and prolonged immobilization are challenges to patients and caretakers. ${ }^{4}$ These complications with osteotomies make hemi-epiphysiodesis an attractive option.

Correction of deformity with reversible hemiepiphysiodesis is much less invasive method. In this angular deformities are corrected by restricting the growth on one side of physis, while allowing growth on non-instrumented side. Gradually, the bone realigns and deformity is corrected. Hemi-epiphyseal arrest can be done using techniques such as stapling, percutaneous drill epiphysiodesis or transphyseal screws. But all these techniques have drawbacks. Stapling technique had drawbacks related to implant failure, migration or breakage of staples and premature closing of physis. The concept of guided growth using 8-plate system have rapidly gained at tention due to low complication rate and encouraging results. 8-plate uses tension band technique, allowing guided growth and doesn't restrict the growth of physis after removal.

In this prospective study, we intended to evaluate the effectiveness of 8-plate guided system in correction of coronal plane deformities around the knee in children regardless of etiology and to assess complications if any. 


\section{Materials and Methods}

The study was carried out on 30 patients with 49 knees from period of June 2015 to March 2017. All patients were followed up for minimum period of 12 months. Pre-operative evaluation was done clinically and radiologically by measuring IMD (Inter malleolar distance), ICD (Intercondylar distance), MAD, TFA, mLDFA (Medial lateral distal femoral angle), MPTA (Medial proximal tibial angle). Full length x-ray was taken from hip to ankle in standing postion. In Blounts disease patients MDA (metaphyseal diaphyseal angle) was measured.

Knee was divided in to 4 equal quadrants and mechanical axis was drawn, valgus as positive and varus as negative. Zone I, the central quadrants, represent plus or minus indicates physiological deformities. Zone II plus or minus indicates symptomatic deformities, may need surgical intervention. Zone III, outside the knee, warrants surgical intervention. If the mechanical axis of the limb crossed the knee joint outside the inner two quadrants of a six quadrant zone it is considered abnormal and offered surgery.

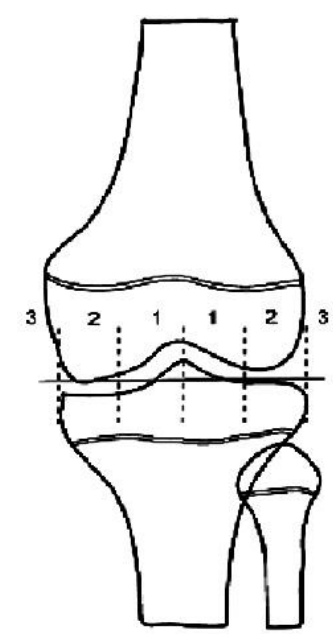

Fig. 1: Radiological zones in knee

Correction was assumed satisfactory if the mechanical axis of the limb shifts to within the inner two central zones. Patients between age 1 and 15 years were included in the study. The age of these patients ranged from 5-14 years with majority between 6-10 years age group. Average age is 9.83 years. In this study 21 patients with 35 knees presented with genu valgum and 9 patients with 14 knees presented with genu varum, 19 patients presented with bilateral deformity, 6 patients presented with left sided deformity and 5 patients presented with right sided deformity.

In this study 7 patients had nutritional rickets, 7 patients were idiopathic, 6 patients had blounts, 6 patients had multiple epiphyseal dysplasia and 4 patients had mucopolysaccharidosis.
Table 1: Aetiological incidence

\begin{tabular}{|l|c|}
\hline Diagnosis & $\begin{array}{c}\text { Number of } \\
\text { Patients }\end{array}$ \\
\hline Nutritional rickets & 7 \\
\hline Idiopathic & 7 \\
\hline Blounts & 6 \\
\hline Med & 6 \\
\hline Mucopolysaccharidosis & 4 \\
\hline
\end{tabular}

Surgical Technique: The surgery was performed under spinal anaesthesia and tourniquet control. The 8-plate was placed over the physis on the centre of the physis and provisionally secured with a small k-wire through a small central hole in the plate, positioning was confirmed by image intensifier. Threaded guide wires were then driven through the two main holes of the plate, aiming to keep the direction of these wires parallel to the physis. The outer cortex drilled using a cannulated drill and a self-tapping $4.5 \mathrm{~mm}$ cannulated screws were passed over the guide wire. These guide wires are removed and screws are finally tightened onto the plate and position is checked in both anteroposterior and later view under image intensifier.

Post-operatively mobilization and full weight bearing allowed immediately. Most of the patients achieved full range of motion by two weeks. Patients were followed up for 4 weeks, 3 months, 6 months, 1 year and 18 months.

In each visit patient were evaluated clinically and radiologically. IMD, ICD, MAD, TFA, mLDFA, MPTA are measured. In Blounts disease metaphyseal diaphyseal angle (MDA) are measured. The desired correction is to attain the mechanical axis within the inner two zones of a six-zone division of an anterioposterior radiograph of the whole limb.In cases, where complete correction was not attained were taken as partially corrected. In cases where no degree of correction or minimal correction was attained were taken as not corrected.

\section{Results}

In our study there are 25 males and 5 female patients. 21 patients with 35 knees presented. In genu valgum group preoperative mean TFA improved from 24.16 to 8.36. Mean MAD improved from 4.26 to 1.09. Rate of correction of TFA is $1.1 /$ month, rate of correction of MAD is $0.22 /$ month. There is statistical significant improvement in all parameters (Table 3).

Table 2: Average rate of correction in 35 knees with genu valgum

\begin{tabular}{|l|c|}
\hline Radiological parameter & $\begin{array}{c}\text { Rate of } \\
\text { Correction }\end{array}$ \\
\hline MAD & 0.22 \\
\hline TFA & 1.1 \\
\hline Mldfa & 0.74 \\
\hline MPTA & 0.16 \\
\hline
\end{tabular}


Table 3: Comparative analysis of parameters preoperatively and last follow-up genu valgum

\begin{tabular}{|l|c|c|c|}
\hline \multicolumn{1}{|c|}{ Preoperative - final follow-up } & t & df & Sig. (2-tailed) \\
\hline IMD & 13.341 & 20 & .000 \\
\hline MAD & 20.448 & 34 & .000 \\
\hline TFA & 19.653 & 34 & .000 \\
\hline MLDFA & -14.064 & 34 & .000 \\
\hline MPTA & 1.976 & 34 & .056 \\
\hline
\end{tabular}

In genu varum group preoperative mean TFA improved from 34.58 to 21.47. Mean MAD improved from 4.92 to 0.63 . Rate of correction of TFA is
$0.77 /$ month, rate of correction of MAD is $0.12 /$ month There is statistical significant improvement in all parameters (Table 5).

Table 4: Average rate of correction in 14 knees with genu varum

\begin{tabular}{|l|c|}
\hline Radiological Parameter & Rate of Correction \\
\hline MAD & 0.12 \\
\hline TFA & 0.77 \\
\hline mLDFA & 0.21 \\
\hline MPTA & 0.78 \\
\hline MDA & 0.76 \\
\hline
\end{tabular}

Table 5: Comparative analysis of parameters preoperatively and last follow-up genu varum

\begin{tabular}{|c|c|c|c|c|c|}
\hline \multirow{3}{*}{$\begin{array}{c}\text { Preoperative - final } \\
\text { follow-up }\end{array}$} & \multicolumn{2}{|c|}{ Paired Differences } & \multirow[t]{3}{*}{$\mathbf{t}$} & \multirow[t]{3}{*}{ df } & \multirow{3}{*}{$\begin{array}{l}\text { Sig. (2- } \\
\text { tailed) }\end{array}$} \\
\hline & \multicolumn{2}{|c|}{$\begin{array}{l}\text { 95\% Confidence Interval of the } \\
\text { Difference }\end{array}$} & & & \\
\hline & Lower & Upper & & & \\
\hline ICD & 6.2750 & 9.7916 & 10.536 & 8 & .000 \\
\hline MAD & 1.5111057 & 2.5603229 & 8.383 & 13 & .000 \\
\hline TFA & 10.1196968 & 16.0945889 & 9.478 & 13 & .000 \\
\hline mLDFA & -5.1645 & 2.1073 & -.908 & 13 & .380 \\
\hline MPTA & -17.0641956 & -9.9643759 & -8.224 & 13 & .000 \\
\hline MDA & 10.9978317 & 16.4221683 & 11.435 & 9 & .000 \\
\hline
\end{tabular}
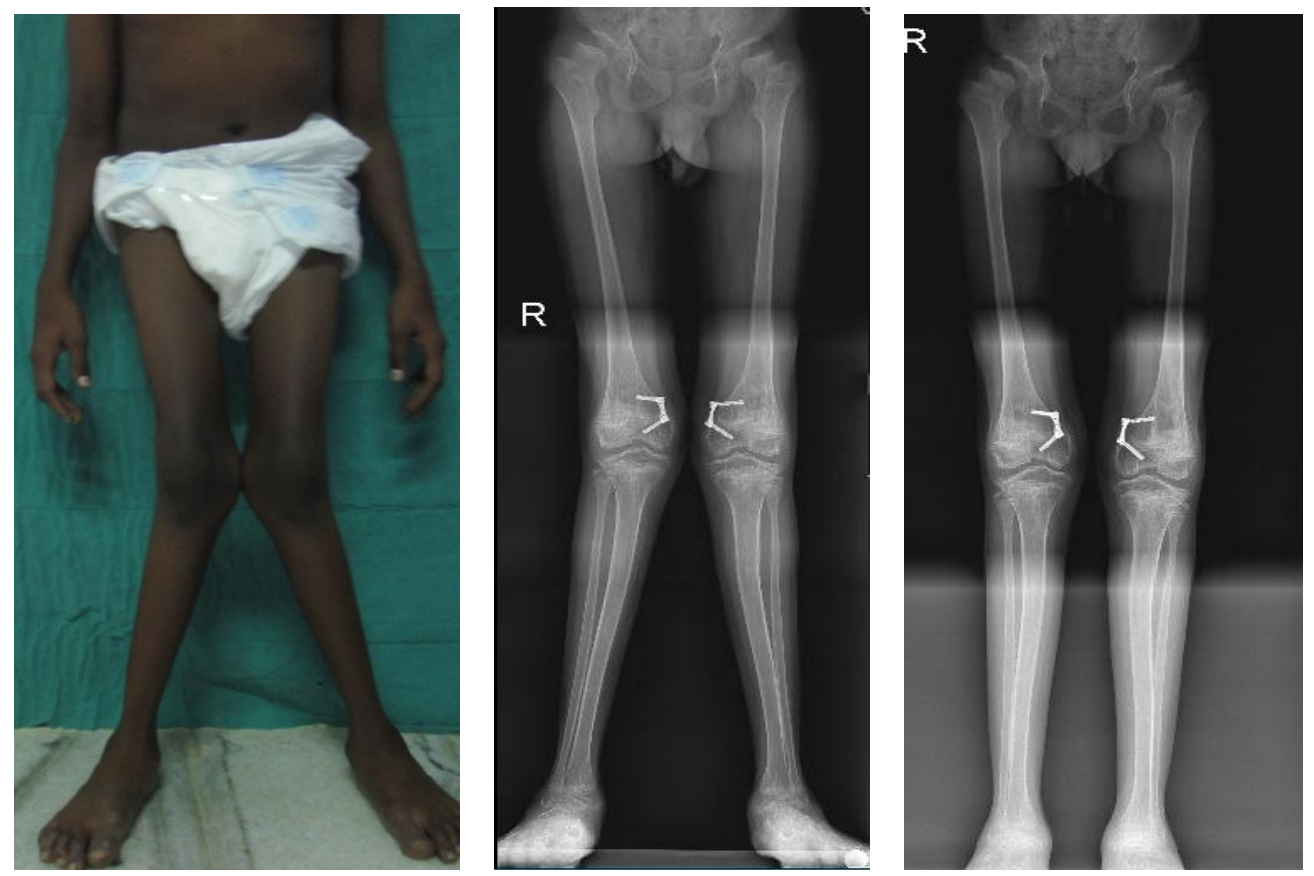

1. Preoperative clinical image; 2. Immediate postoperative radiograph; 3.18 months followup radiograph Fig. 1: 

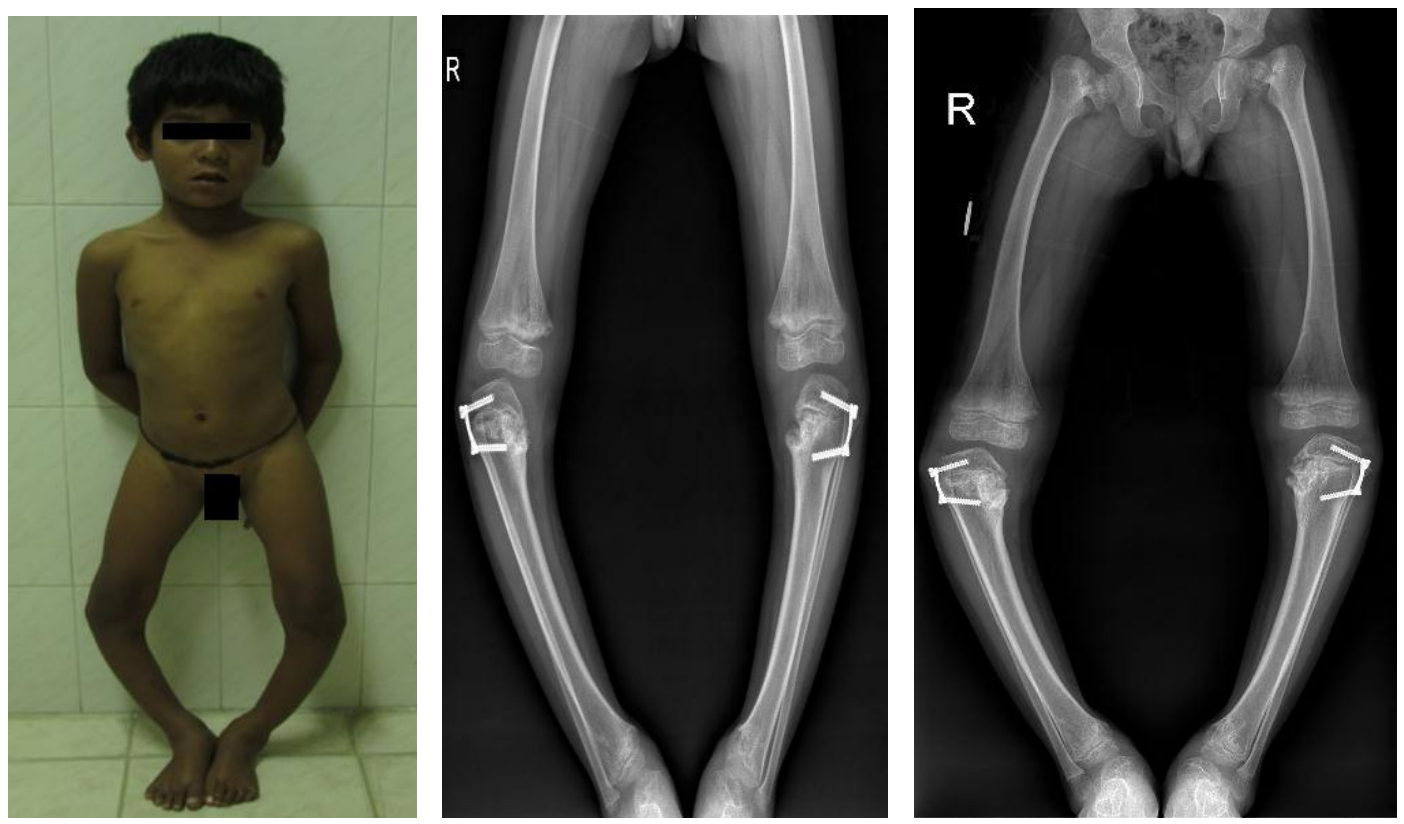

1. Preoperative clinical image; 2. Immediate postoperative radiograph; 3. 18months followup radiograph Fig. 3:

In the present study no case of implant breakage and loosening was encountered. One patient had early physeal closure. Two patients presented with superficial infection at the surgical site, which were resolved after treatment with IV antibiotics and dressing. In this study

Implant removal was done in 11 cases, 2 cases with 2 knees in genu varum and 9 cases with 13 knees in genu valgum after attaining the correction. The average follow-up in genu valgum is 14.95 months and in genu varum is 16.66 months. In genu valgum, MED (Fig. 2) cases got better rate of correction followed by idiopathic and nutritional rickets. In our study in mucopolysaccharidosis type IV-A (Fig. 3) cases the deformity correction was not on par with other cases. In genu varum, idiopathic cases got better results than blounts disease. We observed that unilateral blounts disease got better results than bilateral blounts disease.

However, as our study is a short term study, patients have to be followed till skeletal maturity to look for full correction and any rebound phenomenon.

\section{Discussion}

Angular deformities around the knee cause distorted stress distribution on the knee joint, through Heuter-Volkmann effect hip and ankle also develop impaired growth. ${ }^{3}$ They studied the effect of compression and tension forces on physis. Excessive pressure inhibit the growth of physis. Osteotomies are definitive treatment for correction but, with high rate complications. Less invasive methods like hemiepiphysiodesis is introduced for correction of angular deformities around the knee in children. The concept of epiphysiodesis was developed by Phemister. ${ }^{5} \mathrm{He}$ used this concept in correcting limb length discrepancy. Bowen et al. described
4 patients complained of prominence of 8-plates.There is improvement in gait and cosmetic appearance in all cases. In genu varum 3 cases are considered as fully corrected, 5 cases as partially corrected and one case as not corrected. In genu valgum 10 cases are considered as fully coerrected and 11 cases as partially corrected. hemiepiphysiodesis to correct angular deformity. Reversible epiphysiodesis was proposed by Haas ${ }^{6,7} \mathrm{He}$ demonstrated modulation of physeal growth by surgical intervention. Haas documented inhibition of growth by placing wire loop around distal femoral physis of canine and observed resuming of growth when wire loop was broken.

In 1949, Blount and Clarke presented their results on growth modulation by epiphyseal stapling. Epiphyseal stapling was a better procedure than osteotomies but the problem with staples was that they impose rigid fulcrum within the physis resulting in slow rate of correction and complications like migration and failure. Prolonged presence of this rigid implant may increase the risk of permanent physeal arrest. ${ }^{8}$ Aykut et al. ${ }^{9}$ studied the effect of temporary hemiepiphysiodesis by using staples on growth plate and advised not to disturb the periosteum while placing the staples to prevent physeal bar formation.

Stevens in 2007 introduced the concept of tension band plate for temporary hemiepiphysiodesis. These plates are non-rigid, flexible and have centre of rotation outside the physis acting as a focal hinge. As the physis grows screws toggle in the plate and pivot in the bone and bring gradual correction. The screws tend to diverge as correction proceeds, because of this divergence, only some compression is applied across the physis, that too not constant. When the screws reach maximum divergence there is also facility within the plate to bend. These features reduce the risk of creating 
a permanent physeal tether. These plates decrease the concern of permanent physeal damage by not compressing the physis. The flexible nature of these plates decreases the chance of screw bending and breaking of screws. ${ }^{8,10}$ The speed of correction depends on the nature of growth modulation (8-plate, staples, transphyseal screws), age of the patient and condition of the physis. In pathological physis, growth modulation should be planned early, this prevents pathological loading of the knee. ${ }^{3}$

\section{Conclusion}

Current study reiterates the role of hemiepiphysiodesis in the correction of coronal plane deformities around the knee using 8-plates as an safe and effective procedure. The procedure is simple with minimal surgical trauma. Deformity is corrected gradually sparing neurovascular damage. Complication rate is less with low risk of physeal arrest, screw breakage and migration. Pathological deformities like skeletal dysplasias warrants early intervention for favourable outcome.

\section{References}

1. Salenius P, Vankka E. The development of the tibiofemoral angle in children. J Bone Joint Surg Am. 1975;57:259-61.

2. Heath $\mathrm{CH}$, Staheli LT. Normal limits of knee angle in white children- Genu varum and genu valgum. J Pediatr Orthop. 1993;13:259-62.

3. Stevens PM, Klatt JB. Guided growth for pathologica physes: Radiographic improvement during realignment. $J$ Pediatr Orthop . 2008;28:632-9.

4. Mycoskie PJ. Complication of osteotomies about the knee in children. Orthopedics. 1981;4(9):1005-1015.

5. Phemister DB. Operative arrestment of longitudinal growth of bone in the treatment of deformities. J Bone Joint Surg. 1933;15:1-15.

6. Haas SL. Retardation of bone growth by a wire loop. $J$ Bone Joint Surge AM. 1945;27:25-36.

7. Haas SL. Mechanical retardation of bone growth. J Bone Joint Surge AM. 1948; 39: 506-12.

8. Sakti Prasad Das, S Pradhan, PK Sahoo et al. Our experience with correction of angular deformities of knee by flexible figure of 8-plate hemiepiphysiodesis. IJPMR Jun 2012; Vol 23(2):68-73.

9. Aykut US, Yazici M, Kandemir U, et al. The effect of temporary hemiepiphyseal stapling on the growth plate: a radiologic and immunohistochemical study in rabbits. $J$ Pediatr Orthop 2005;25:336-41.

10. Ballal MS, Bruce CE, Nayagam S. Correcting genu varum and genu valgum in children by guided growth: Temporary hemiepiphysiodesis using tension band plates. J Bone Joint Surg Br. 2010;92:273-6. 